\title{
Pérdida y fragmentación del bosque nativo en la cuenca del río Aysén (Patagonia-Chile) durante el siglo $\mathrm{XX}^{1}$
}

\author{
Gustavo Bizama², Fernando Torrejón², Mauricio Aguayo², María D. \\ Muñoz ${ }^{2}$, Cristian Echeverría ${ }^{3}$ y Roberto Urrutia ${ }^{2}$
}

\begin{abstract}
RESUMEN
En este trabajo se estimó la pérdida y fragmentación de bosque nativo en la cuenca del río Aysén, ocurrida entre los años 1900 y 1998, debido principalmente a incendios forestales producto de la colonización del territorio. En la reconstrucción de la cobertura boscosa fueron de gran utilidad distintos registros históricos y el sistema de información geográfica (SIG ArcView 3.2). Se aplicaron diferentes índices del paisaje (área, densidad y tamaño del fragmento, área núcleo total, distancia euclidiana, índices de forma y de agregación, y longitud de borde total), evaluándose los cambios en los patrones de distribución del bosque nativo entre 1900 y 1998. Se reconoció que el bosque perdido fue reemplazado mayormente por praderas, evidenciándose una pérdida aproximada del $23 \%$, de bosque nativo, identificándose además un aumento de fragmentos menores a 100 ha, y la disección de estos debido a los incendios generados durante la colonización. Sin embargo, dichos fragmentos presentarían una alta conectividad, situación que fue corroborada a través de diferentes índices de paisaje.
\end{abstract}

Palabras clave: Incendios forestales, colonización, fragmentación de bosque, Patagonia, Chile.

\begin{abstract}
This work estimates the loss and fragmentation of native forest in the watershed of the river Aysén during the $20^{\text {th }}$ century, as a result of clearance fires induced by land settlers. In order to generate the reconstruction of the native forest cover, several documentary records and GIS ArcView 3.2 were used. Different indexes of landscape changes were applied (area, density and size of the fragment, core area, euclidean distance, shape and aggregation index, and edge length) to estimate the distribution of the native forest between 1900 and 1998, which indicate that the main replacement of the forest was by prairies. A loss of approximately $23 \%$ of native forest was registered, also an increase in the number of forest fragments $(<100 \mathrm{ha})$, as a result of the settlement process. Howerer, the increasing number of fragments has a high connectivity, wich was corroborated using different landscape indexes.
\end{abstract}

Key words: Forest fires, settling, fragmentation of forest, Patagonia, Chilean.

1 La presente investigación ha sido desarrollada y
financiada en el marco de los proyectos FONDECYT
$\mathrm{N}^{\circ} 1070508, \mathrm{~N}^{\circ} 1070511$ y $\mathrm{N}^{\circ} 1060633$. Artículo
recibido el 23 de agosto de 2010 , aceptado el 2 de
junio de 2011 y corregido el 30 de junio de 2011.
2 Centro de Ciencias Ambientales EULA-Chile, Uni- 
La fragmentación del hábitat ha sido reconocida como una de las principales amenazas para los ecosistemas de todo el mundo (Armenteras et al., 2003; Lindenmayer \& Fischer, 2006). El concepto de fragmentación puede ser definido como la transformación de un bosque continuo en unidades más pequeñas y aisladas entre sí, cuya área resultante es mucho menor a la del bosque original (Andrén, 1994; Bustamante y Grez, 1995). La fragmentación consiste en tres fenómenos que se producen paralelamente: i) división de hábitat en porciones menores y discontinuas, ii) disminución del tamaño de los hábitats remanentes y iii) aislamiento progresivo de los parches dentro de una matriz generalmente hostil (Forman \& Godron, 1986; Fahrig, 1997; Fahrig, 2003; Pauchard et al., 2006); lo último debido al alto contraste, en términos de su composición o estructura, entre los fragmentos y el paisaje circundante que representa la matriz (Saunders et al., 1991; Aguayo, 2001).

Existen diversas causas que darán origen a la fragmentación, entre las que se encuentran las de origen natural, sin embargo, como lo señalan Bustamante y Grez (1995) "Ios científicos han llegado a un consenso, determinando que el principal agente perturbador es el hombre" (Bustamante y Grez, 1995: 58). En el mismo sentido, Aguayo (2001) indica que el hombre ha provocado la transformación del paisaje natural a través de la intensificación del uso del suelo para su desarrollo económico. Estos cambios de uso, asociados a la expansión agrícola y explotación forestal, han acelerado la pérdida, fragmentación y degradación de hábitat silvestres en el último siglo, proyectándose esta tendencia hacia las próximas décadas (Fahrig, 2003).

De esta manera, la fragmentación del bosque desencadena una serie de procesos que deterioran el hábitat, los que pueden estar asociados a efectos de borde, generando cambios en las condiciones abióticas del fragmento (Chen et al., 1995; Laurance, 2000; López-Barrera et al., 2004; LópezBarrera et al., 2005; Ries et al., 2004; Zeng $\& W u, 2005)$. Otro efecto es el aislamiento progresivo de las poblaciones que habitan estos fragmentos, que dependerá tanto de la conectividad de los fragmentos como de la adversidad de la matriz que los rodea
(Fahrig \& Merriam, 1994; Burel y Baudry, 2002; Santos y Tellería, 2006; Fletcher et al., 2007).

Los bosques templados de Chile no se encuentran ajenos a las perturbaciones antrópicas que también causan la fragmentación (Aguayo, 2001). Chile posee el área de bosques templados más grande de Sudamérica, y más de la mitad del área total de bosques templados en el hemisferio austral (Donoso, 1993). Según Armesto et al. (1997) la mayor parte de estos bosques se ubican de forma continua entre el río Maule ( $\left.35^{\circ} \mathrm{S}\right)$ y Tierra del Fuego $\left(55^{\circ} \mathrm{S}\right)$, aunque en la actualidad dichas formaciones vegetales se encontrarían de forma cada vez más intermitente. De acuerdo con el estudio realizado por CONAF et al. (1999), en 1997 la superficie cubierta por el bosque templado abarcaba aproximadamente 13,4 millones de hectáreas en el país. Este bioma ha sido clasificado como un Hotspot para la conservación, debido a su alta diversidad biológica y endemismo (Armesto et al., 1997; Myers et al., 2000; Quintanilla, 2001).

Actualmente el área de los bosques templados chilenos se ha reducido en una magnitud similar a la disminución experimentada durante la última edad glacial, debido al impacto humano ocurrido en los últimos 200 años (Armesto et al., 1994). Dentro de las principales causas de la disminución y/o destrucción del bosque nativo está la producción de leña y carbón, producción de chips y reemplazo de este bosque por plantaciones forestales (Simonetti y Armesto, 1991; San Martín y Donoso, 1996). Los incendios forestales de origen humano así como la sustitución del bosque por praderas han sido otros factores que han incidido fuertemente en la pérdida de este tipo de cobertura vegetal (Lara et al., 2000).

Una de las zonas de Chile afectada por las perturbaciones antrópicas antes mencionadas fue la cuenca del río Aysén, caracterizada por las formaciones vegetales de bosque siempreverde y de turberas, estepa patagónica y bosque patagónico (Gajardo, 1994). En esta zona, colonizada a partir de inicios del siglo XX, habrían ocurrido los más grandes incendios de bosques registrados en Chile, consumiéndose alrededor de 
3.000.000 ha (Grosse, 1955; Grosse, 1974; Cunill, 1979; Gajardo, 1994; Ortega \& Brüning, 2004; Donoso \& Otero, 2005; Martinic, 2005; Otero, 2006; Quintanilla, 2008; Torres-Gómez et al., 2009). Estos grandes incendios ocurridos en la cuenca del río Aysén, durante el siglo XX, podrían haber sido determinantes en la pérdida de conectividad de sus bosques, debido a que habrían generado una alta pérdida y fragmentación de la cobertura vegetal original.

En consecuencia dado que no existen estudios específicos que permitan cuantificar la pérdida y fragmentación del bosque nativo en la cuenca del río Aysén, el presente trabajo busca reconocer y evaluar los cambios de los patrones de distribución del bosque nativo entre los años 1900 y 1998.

\section{Materiales y métodos}

\section{Definición del área de estudio}

La cuenca del río Aysén pertenece a la jurisdicción de la Región del General Carlos Ibáñez del Campo, Chile, entre los $45^{\circ} 00^{\prime}$ $46^{\circ} 16^{\prime}$ Sur y $71^{\circ} 20^{\prime}-73^{\circ} 00^{\prime}$ Oeste, abarcando una superficie de 1.144.145 ha (Figura $\left.N^{0} 1\right)$. Sus principales tributarios son: el río Mañiguales, Simpson, Blanco y río de Los Palos (IGM, 1984). La zona se caracteriza por un fuerte gradiente de precipitación oesteeste, el que varía desde $3.000 \mathrm{~mm}$ promedio anual en la costa oeste (Puerto Aysén, 45 $24^{\prime}$ Sur-72 $2^{\circ} 42^{\prime}$ Oeste), hasta llegar a $1.164 \mathrm{~mm}$ en Coyhaique $\left(45^{\circ} 34^{\prime}\right.$ Sur-72 ${ }^{\circ} 04^{\prime}$ Oeste) (Miller, 1976). En la vertiente oriental, Ios valores de las precipitaciones decrecen hasta

Figura $\mathrm{N}^{0} 1$

Ubicación y coberturas del área de estudio, cuenca del río Aysén

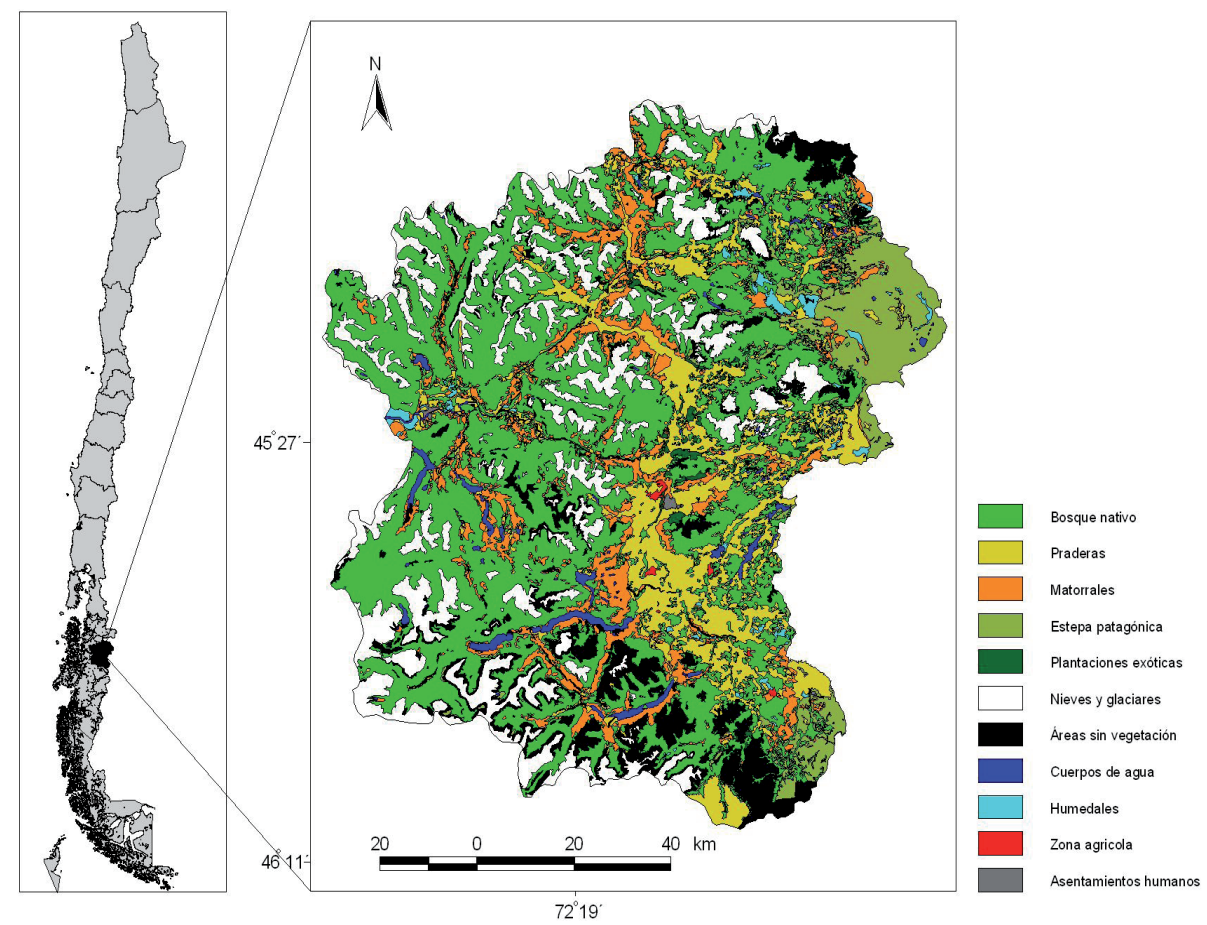

Fuente: Modificado de CONAF et al., 1999. 
los $621 \mathrm{~mm}$ anuales en Balmaceda $\left(45^{\circ}\right.$ 55' Sur- $71^{\circ} 41^{\prime}$ Oeste), registrándose los máximos de agua caída en los meses de mayo, julio y agosto (DGA, 2004). La explotación económica mercantil de la cuenca del río Aysén se inició recién en la década de 1900, con la concesión de grandes extensiones de tierras otorgadas a compañías ganaderas particulares, y la llegada de los primeros colonos a la zona. Este proceso se intensificó hacia 1930 , como resultado del aumento de la ocupación espontánea del hábitat que hasta entonces se encontraba sin intervención humana (Ibáñez, 1972-1973; Cunill, 1979; Martinic, 2005).

\section{Reconstrucción del bosque nativo histórico}

Se realizó un análisis espacial de la cuenca del río Aysén mediante el uso de la base de datos cartográfica del catastro y evaluación de los recursos vegetacionales nativos de Chile (CONAF et al., 1999). Esta base de datos fue manipulada a través de un Sistema de Información Geográfica, utilizando los software ArcView 3.2 (ESRI, 1999) y FRAGSTATS 3.3 (McGarigal et al., 2002). La antigua cobertura vegetal existente hacia 1900, que habría estado compuesta principalmente por bosque nativo, fue reconstruida reclasificando las praderas, zonas agrícolas, plantaciones exóticas y asentamientos humanos, debido a que estos nuevos usos de suelo habrían correspondido originalmente a áreas boscosas. Paralelamente se reconstruyó el paisaje original del área de estudio, utilizándose registros documentales consistentes en descripciones bibliográficas, cartografía, iconografía y fotografías históricas (Bolós, 1992). En este contexto, fue relevante la fotointerpretación supervisada de una serie de fotografías aéreas del año 1943 (Vuelo Trimetrogon, escala 1:60.000). Complementariamente, durante los periodos estivales de los años 2007-2009, se realizó trabajo de campo consistente en la inspección visual de zonas representativas de la cuenca del río Aysén, cuya finalidad fue verificar el cambio de uso de suelo histórico mostrado por la evidencia documental.

\section{Índices del paisaje}

Para realizar el análisis del patrón de distribución del bosque nativo en el área de estudio, se utilizaron diferentes índices de paisaje. Estos fueron seleccionados a partir de una serie de estudios previos relacionados con la fragmentación forestal: i) área del fragmento $^{4}$, ii) área núcleo total ${ }^{5}$, iii) densidad de fragmentos por cada $100 \mathrm{ha}^{6}$, iv) distancia euclidiana $^{7}$, v) índice de forma ${ }^{8}$, vi) índice de agregación ${ }^{9}$, vii) tamaño del fragmento más grande $^{10}$ y viii) longitud total del borde ${ }^{11}$; todos ellos obtenidos mediante el software FRAGSTATS 3.3 (Aguayo, 2001; Staus et al., 2002; Cayuela et al., 2006; Echeverría et al., 2006; Echeverría et al., 2007; Pauchard et al., 2006; McGarigal et al., 2002).

\section{Resultados y discusión}

La cuenca del río Aysén abarca una superficie total de 1.144.145 ha, distribuidas en 2.262 fragmentos; de estos, 439 corresponden a bosque nativo, presentando una superficie de 521.463 ha, equivalente a un 45,5\% del área total de la cuenca. El 54,5\% restante de la cuenca se distribuyen en diferentes tipos de coberturas (Cuadro $\mathrm{N}^{\circ} 1$ ).

4 Corresponde al tamaño del área de cobertura de cada fragmento de bosque nativo, en hectáreas.

5 Equivale a la suma de las áreas núcleo de cada fragmento de bosque nativo, las que fueron calculadas una vez definidas las diferentes profundidades de efecto borde, que en este caso se establecieron a $100 \mathrm{~m}, 300 \mathrm{~m}$ y $500 \mathrm{~m}$.

6 Índice que se obtiene dividiendo el número total de fragmentos por cada 100 hectáreas.

7 Se obtiene calculando la distancia más corta, en línea recta, entre el borde del fragmento focal y el borde de su vecino más cercano.

8 Este índice puede tomar valores mayores o iguales a 1. El valor 1 se alcanza cuando los fragmentos son compactos o regulares, tales como un cuadrado o un círculo. Cuando el valor 1 es sobrepasado significa que los fragmentos tienden a la irregularidad (McGarigal et al., 2002).

9 Se refiere al porcentaje de "adyacencias comunes" de una determinada clase de uso de suelo (en el caso del presente estudio, bosque nativo) (McGarigal et al., 2002).

10 Corresponde al área del parche de mayor tamaño dividido por el área total del paisaje (área de estudio). Este valor es multiplicado por 100 para convertirlo en porcentaje.

${ }^{11}$ Es la suma de los perímetros de todos los parches de bosque nativo, expresado en metros. 
Cuadro $\mathrm{N}^{\circ} 1$

Usos del suelo actual de la cuenca del río Aysén

\begin{tabular}{|l|r|c|c|}
\hline \multicolumn{1}{|c|}{ Tipos de uso/cobertura } & Superficie (ha) & Superficie (\%) & Número de fragmentos \\
\hline Bosque nativo & $521.463,4$ & 45,5 & 439 \\
Matorrales & $102.028,0$ & 8,9 & 410 \\
Zona agrícola & $1.604,5$ & 0,1 & 10 \\
Humedales & $10.998,0$ & 0,9 & 92 \\
Áreas sin vegetación & $90.200,1$ & 7,8 & 324 \\
Praderas & $158.490,2$ & 13,8 & 431 \\
Estepa patagónica & $76.697,5$ & 6,7 & 157 \\
Cuerpos de agua & $17.718,9$ & 1,5 & 191 \\
Nieves y glaciares & $157.548,2$ & 13,7 & 147 \\
Plantaciones exóticas & $6.109,2$ & 0,5 & 50 \\
Asentamientos humanos & $1.287,4$ & 0,1 & 11 \\
Total & $1.144 .145,3$ & 100 & 2.262 \\
\hline
\end{tabular}

Fuente: Datos modificados de CONAF et al., 1999.

El bosque nativo del área de estudio se encuentra inserto en una matriz donde predominan matorrales, nieves, estepa patagónica, además de praderas, y como se puede apreciar en la Figura $\mathrm{N}^{\circ} 1$ este último tipo de cobertura prácticamente separa los bosques, pudiendo actuar como barrera para el desplazamiento de la fauna asociada a estos ecosistemas. Las especies que se verían más afectadas son las multihábitat, especialistas y aquellas cuya capacidad de dispersión se ve afectada negativamente por las praderas (Burel y Baudry, 2002). Estas praderas, de origen artificial, fueron creadas por incendios provocados para habilitar terrenos que serían destinados a la ganadería extensiva, de esta manera, los antiguos bosques existentes en el área de estudio fueron eliminados mediante el roce, para luego sembrar el terreno despejado con pasto miel (Paspalum dilatatum) (Keller, 1948; Grosse, 1955; Grosse, 1974; Otero, 2006; Quintanilla, 2008). Dichos incendios se incrementaron a medida que crecía la colonización del territorio, favorecida por la ley $N^{\circ} 4.855$ de tierras y colonización de 1930 (Sepúlveda, 1931; Ibáñez, 19721973). En la actualidad, tal como se observó in situ, en muchas de estas praderas aún es posible reconocer, en mayor o menor medida, restos de enormes troncos quemados, vestigios de los incendios generados hace ya décadas.

El caso de los matorrales merece una atención especial, pues si bien autores como Segura et al. (1998) sugieren que el crecimiento y formación de matorrales sería, en muchos casos, la resultante de la quema de bosques, la evidencia histórica analizada, incluida la fotointerpretación (Vuelo Trimetrogon de 1943) demostraría la existencia de dichas formaciones vegetales con anterioridad a la ocurrencia de incendios en el área (Simpson, 1875; Steffen, 1909; Pomar, 1923; Reiche, 1934). En efecto, la mayoría de estos matorrales crecían en suelos húmedos y/o pantanosos, donde las escasas especies arbóreas no llegaban a constituir bosques. De acuerdo a Pomar (1923) estas formaciones vegetales estaban conformadas principalmente por quila (Chusquea chilensis), especie que habría sido usada como forraje para ganado, razón por la cual en muchos casos habrían sido preservadas (Sepúlveda, 1931). 
Por otra parte, los resultados del presente estudio indican que la superficie del bosque nativo actual en la cuenca del río Aysén es de 521.463 ha. De acuerdo a la información proporcionada por la evidencia histórica bibliográfica e iconográfica, se calcula que hacia 1900 la superficie del bosque nativo habría alcanzado alrededor de las 675.519 ha. El alto nivel de resolución temporal y la calidad testimonial de los registros históricos empleados (Simpson, 1875; Steffen, 1909; Pomar, 1923; Sepúlveda, 1931; Grosse, 1955), permiten asumir que, a inicios del siglo XX, el área de estudio presentaba condiciones de "alta virginidad", encontrándose además prácticamente despoblada, lo que facilitó la elaboración reconstructiva efectuada. Esta reconstrucción estaría corroborada por la presencia de innumerables restos de troncos calcinados dispersos en muchas de las áreas que paulatinamente fueron convertidas en praderas por la acción colonizadora del territorio de Aysén durante el siglo XX12.

Al compararse las cifras ya señaladas (675.519 ha de bosque nativo ca. $1900 \mathrm{v} / \mathrm{s}$ las 521.463 ha de bosque nativo en 1998), se puede establecer una pérdida aproximada de 154.056 ha, equivalentes a un $23 \%$ de bosque original (Figura $\mathrm{N}^{\circ} 2$ ). A la vez, se genera una disminución del tamaño promedio de los fragmentos, de un $68 \%$ aproximadamente, y un aumento en la densidad de fragmentos del bosque nativo de 1,8 a 4,3 por cada 100 ha (Cuadro $N^{\circ} 2$ ); es decir, se incrementa el número de fragmentos en un $119 \%$.

12 Trabajo de campo 2007-2009.

Figura $\mathrm{N}^{\circ} 2$

Cambios de los patrones de distribución del bosque nativo de la cuenca del río Aysén
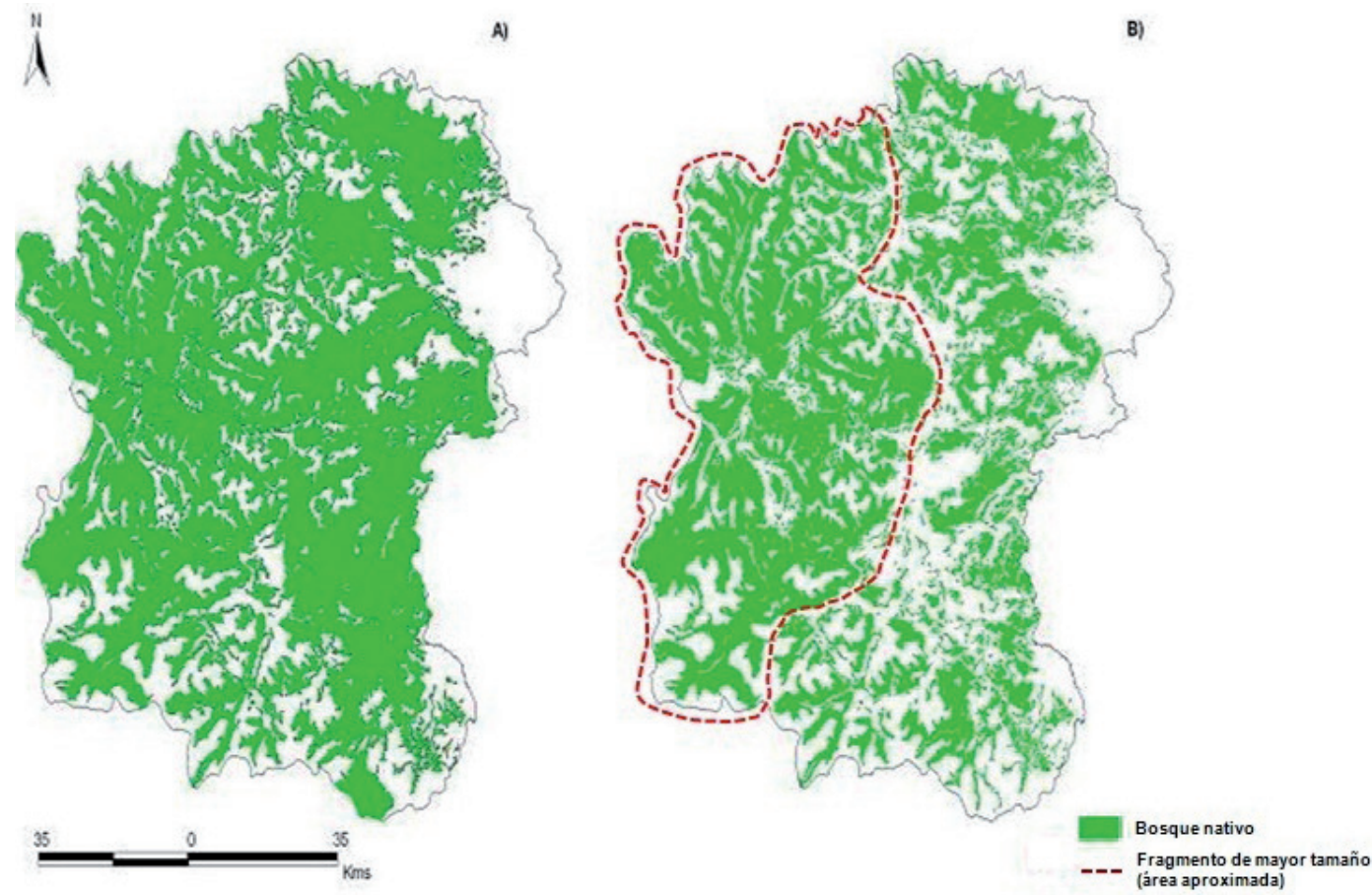

Nota: A) año ca. 1900 y B) 1998.

Fuente: Elaboración propia. 
Cuadro $\mathrm{N}^{\circ} 2$

Cambios en el patrón espacial del bosque nativo de la cuenca del río Aysén (ca. 1900-1998)

\begin{tabular}{|l|c|c|}
\hline \multicolumn{1}{|c|}{ Índices de paisaje } & "Bosque histórico" ca. 1900 & "Bosque actual" 1998 \\
\hline Área promedio de los fragmentos (ha) & $3.691,3[0,81-659.443,6]$ & $1.187,8[0,81-280.630,9]$ \\
Tamaño del fragmento más grande (\%) & 97,6 & 53,8 \\
Densidad de fragmentos (n/100 ha) & 1,8 & 4,3 \\
Longitud total del borde (m) & 13.091 .220 & 15.610 .500 \\
Distancia euclidiana (m) & $338,2[180-3.057,3]$ & $346,1[180-7.029,8]$ \\
\hline
\end{tabular}

Nota: Los índices, área promedio del fragmento (ha) y distancia euclidiana (m), corresponden a los valores promedio, y los contenidos entre corchetes a los valores mínimos y máximos respectivamente.

Fuente: Elaboración propia.

Es importante destacar aquí que la fragmentación de los bosques de la zona se habría debido principalmente a incendios forestales provocados durante el proceso de colonización espontánea del área, tal como lo evidencian las fuentes históricas utilizadas (Pomar, 1923; Sepúlveda, 1931; Grosse, 1955). Si bien otras actividades productivas tales como la extracción de leña y manufactura de productos madereros, también pudieron haber incidido en la pérdida de bosques (Gómez-Lobo, 2005; Martinic, 2005), esta habría sido comparativamente menor a la que en su momento generaron los incendios. En este mismo sentido la herbivoría generada por el ganado exótico habría sido marginal en los cambios de los patrones de distribución del bosque nativo, pues los grandes hatos de ganado ovino, bovino y caballar fueron introducidos paulatinamente tras la quema de los bosques. Sin embargo, en la actualidad esta variable ecológica contribuiría a mantener los cambios en los patrones de distribución del bosque nativo (Quintanilla, 2008).

Entre 1903 y 1905, el Estado de Chile otorgó, tanto a particulares como a sociedades anónimas, las primeras concesiones de tierras en la cuenca del río Aysén (Coyhaique, Ñirehuao y Mañiguales). Se debe destacar que hacia 1902 la región interior se encontraba "prácticamente virgen" (Ibáñez, 1972-1973: 299). Posteriormente, a partir de 1930, se iría desarrollando un proceso de colonización espontánea de los terrenos adyacentes a las concesiones del las grandes compañías ganaderas allí establecidas (Sociedad Ganadera de los Tres Valles, Sociedad del Valle del Río Cisnes y Sociedad Industrial de Aysén-SIA) (Pomar, 1923; Ibáñez, 1972-1973). Debido a que esta última sociedad se adjudicó los mejores territorios para la ganadería llamados erróneamente "praderas naturales", pero que en realidad correspondían a estepa patagónica, los colonos que ocuparían los terrenos restantes se verían obligados a "limpiar" los bosques vírgenes mediante roces, para así obtener tierras aptas para desarrollar su principal actividad productiva, basada en la ganadería de lanares, caballares y vacunos. De acuerdo a Martinic (2005), en aquellos tiempos el fuego fue el mejor aliado de los colonos, debido a que estos no poseían medios para la explotación maderera del bosque, así la inmensa pérdida de cobertura vegetal fue producto de los grandes incendios, tal como lo señala el mismo autor: "fue el duro precio que ha debido pagarse para la conquista territorial de la región de Aysén" (Martinic, 2005: 308) (Figura No 3A). Fueron estos incendios generados durante la colonización del territorio los que provocaron una disección de la cobertura vegetal original, resultando trascendentales en el patrón de distribución que presenta el bosque nativo en la actualidad (Figura No 3B). 
Figura $\mathrm{N}^{\circ} 3$

A) Bosques incendiados dentro del área estudio, valle Mañihuales. B) Vestigios de una antigua quema, camino a laguna Thompson, Coyhaique

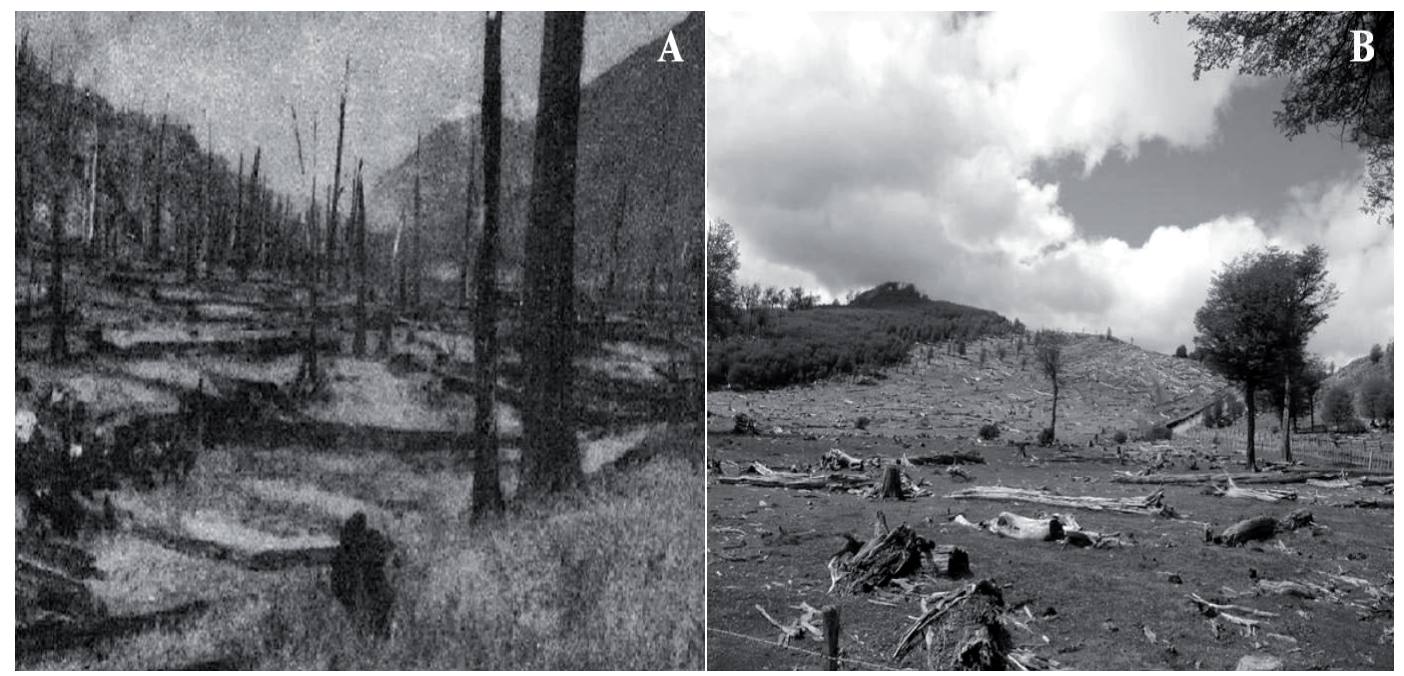

Fuente: Grosse, 1955 y Trabajo de campo 2009, fotografía de F. Torrejón.

Es interesante señalar que el único antecedente documentado referido a incendios forestales en la cuenca del río Aysén, ocurridos previos a la colonización, corresponde al registro del fuego accidental generado por individuos de la sección exploradora de Fischer y Bronsart (expedición de Steffen al río de Aysén, 1896), quienes el 27 de febrero de 1897, provocaron un incendio de proporciones en las cercanías del actual Coyhaique (Steffen, 1909). Sin embargo, Steffen (1909) reconoce la existencia de otras quemas antiguas en el área de estudio, pero no tiene certeza sobre su origen, señalando que probablemente dichas quemas fueron originadas "por algunos colonos que, desde el lado arjentino, se habian internado en el valle en busca de terrenos útiles para la ganadería" (Steffen, 1909: 122). Es muy dudoso que las quemas de bosques datadas hacia fines del siglo XIX hayan sido provocadas por tehuelches, pues si bien estos indígenas usaban el fuego como una herramienta auxiliar en la caza de guanacos (Lama guanicoe) y ñandúes (Pterocnemia pennata), esta actividad cinegética (el chaco) se realizaba exclusivamente en la estepa pata- gónica (Musters, 1911). Los demás incendios documentados, ya en el siglo XX, fueron efectuados por la Sociedad Industrial de Aysén (SIA), para realizar "arreglos particulares"; la más importante de estas quemas fue la provocada también en las cercanías de Coyhaique, involucrando 550 ha. Otro de estos incendios ocurrió en Puerto Aysén, donde se quemaron 200 ha; y el más pequeño en el sector del Balsadero, alcanzando solo 50 ha (Pomar, 1923). Durante este proceso de quemas se limpiaron y sembraron, con pasto miel, alrededor de 800 hectáreas (Pomar, 1923).

Al compararse la distribución del número de fragmentos, por clase de tamaño, de bosque nativo presente entre los años 1900 y 1998, se puede observar un aumento en el número de fragmentos menores a 100 ha. Para el bosque nativo histórico se determinaron 158 fragmentos, mientras que para el bosque nativo actual, se identificaron 320 , lo que implica un aumento del $101 \%$ en el número de fragmentos. Un aumento mucho menor se registró para el resto de las clasificaciones (Figura $\mathrm{N}^{\circ} 4$ ). 
Figura $\mathrm{N}^{\circ} 4$

Cambios en los patrones de distribución del tamaño de los fragmentos de bosque nativo entre los años 1900 y 1998

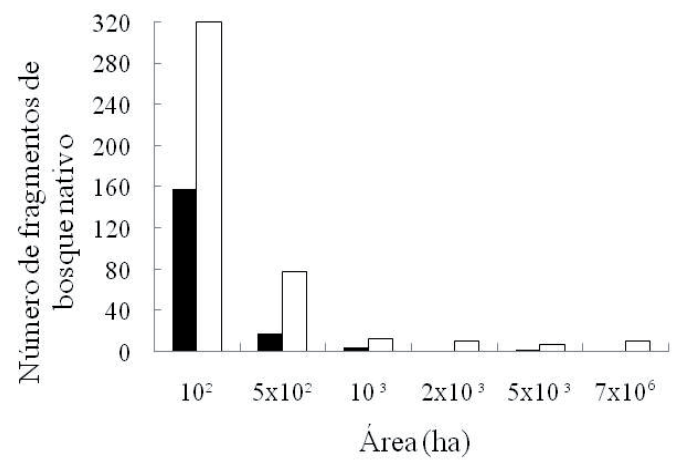

Nota: año 1900 (en negro) y 1998 (en blanco). Fuente: Elaboración propia.

En el mismo contexto, autores como Armenteras et al. (2003), Cayuela et al. (2006) y Altamirano et al. (2007), señalan que la predominancia de fragmentos de pequeño tamaño sugeriría un alto nivel de fragmentación del bosque, situación que ocurre en la cuenca del río Aysén. Sin embargo, considerando el tiempo transcurrido, de casi 100 años, en la comparación realizada, el incremento en la cantidad de fragmentos no sería sustancial, debido a que el bosque nativo actual de la zona aun presenta una alta continuidad, donde el fragmento de mayor tamaño abarca aproximadamente 280.630 ha, equivalentes a un $53,8 \%$ del total de bosque nativo presente en el área de estudio (Cuadro $N^{\circ} 2$ ). Cabe señalar que este gran fragmento de bosque nativo se encuentra ubicado en el flanco occidental de la cuenca del río Aysén (Figura $\mathrm{N}^{\circ}$ 2B), zona que por sus condiciones ambientales -alta pluviosidad, accidentada geografía $y$, en general, el alto grado de aislamientotuvo una colonización marginal, por lo que no se vio afectada por los grandes incendios ocurridos entre las décadas del 1940 y 1950 (Grosse, 1955; Grosse, 1974; Martinic, 2005; Quintanilla, 2008). La conservación de dicho fragmento sería de vital importancia, debido que al presentar una mayor superficie $y$ perímetro, permitiría el mantenimiento de grandes poblaciones, un menor efecto de borde y mayor dificultad de ser colonizada por especies invasoras (Sepúlveda et al., 1997). Por el contrario en el sector oriental (ecotono bosque-estepa), predominan los fragmentos de pequeño tamaño, que serían más vulnerables al efecto de borde; aún así, estos fragmentos son muy importantes para la conservación, debido a que son utilizados como puntos de paso en el desplazamiento de la avifauna local; pudiendo servir a la vez como hábitats temporales para algunas de estas especies (Burel y Baudry, 2002; Lindenmayer \& Fischer, 2006).

No obstante los incendios históricos que causaron la disección del bosque, la totalidad de los actuales fragmentos no presentarían una alta pérdida de conectividad física, cuestión que se evidencia en el índice de agregación, con un valor de 95,7\% para el año 1900 y de 93,3\% para el año 1998. Esto sería corroborado por la diferencia, bastante sutil, de los resultados de la distancia euclidiana del vecino más cercano (DVC) (Cuadro $N^{\circ}$ 2), donde el promedio de los fragmentos del bosque nativo histórico es de una distancia de $338 \mathrm{~m}$, mientras que para el bosque nativo actual alcanza $346 \mathrm{~m}$. Sin embargo, se puede observar que el fragmento más aislado, presentó un notable aumento correspondiente a un $115 \%$, es decir, desde los $3.057 \mathrm{~m}$ que tenía en el año 1900, se incremento hasta los $7.029 \mathrm{~m}$ en el año 1998.

Si bien el valor de los índices usados para caracterizar y cuantificar la fragmentación de un paisaje varía dependiendo de la escala o resolución a la que fueron obtenidos los datos (Pauchard et al., 2006), la posibilidad de detectar pequeños fragmentos a una mayor resolución no se constituiría en una limitante; esto debido a que los índices utilizados para evaluar la conectividad no cambiarían drásticamente a menos que las distancias entre los fragmentos, calculadas desde el borde, varíen de forma considerable. En consecuencia, la aparición de nuevos fragmentos sería proporcional para los distintos tipos de coberturas de suelo, compensándose las distancias entre fragmentos del mismo tipo, manteniéndose de esta manera la conectividad.

En cuanto al índice de forma y a su relación con aquellos fragmentos de bosque nati- 
vo menores a 100 ha, estos conservarían una forma más bien compacta y regular, sin apreciarse un cambio sustancial entre el bosque nativo histórico y el bosque nativo actual. Dado este resultado es posible señalar, tal como lo indican Bustamante y Grez (1995) y Aguayo (2001), que no existiría un marcado efecto de borde en los fragmentos del bosque nativo actual (Figura $N^{\circ} 5$ ).

\section{Figura $\mathrm{N}^{\circ} 5$}

Índice de forma y su relación con el tamaño de los fragmentos menores a 100 ha

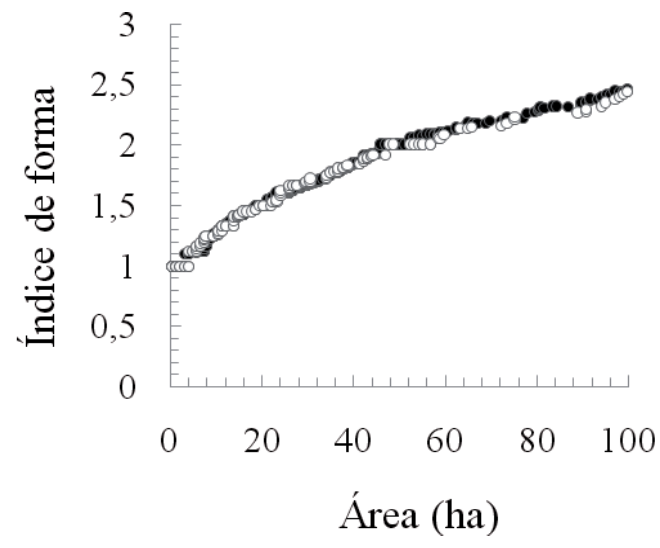

Nota: ( o ) "bosque histórico" (ca. 1900) y en ( "bosque actual" (1998).

Fuente: Elaboración propia.

Las áreas que no se encontrarían afectadas por el efecto borde, denominadas "áreas núcleos" (Pauchard et al., 2006), evidenciaron una drástica disminución en hectáreas, pues los resultados muestran una notoria pérdida de bosque a diferentes profundidades de efecto borde $(100 \mathrm{~m}, 300 \mathrm{~m}$ y $500 \mathrm{~m})$. Esta pérdida fue similar tanto en el año 1900 como en 1998, lo que se explica debido a la escasa variación en la forma de los fragmentos de bosque nativo ya señalada (Figura $\mathrm{N}^{\circ}$ $6)$.
Figura $\mathrm{N}^{\circ} 6$

Comparación de las áreas núcleos totales a diferentes profundidades de efecto borde $(100 \mathrm{~m}, 300 \mathrm{~m}$ y $500 \mathrm{~m})$

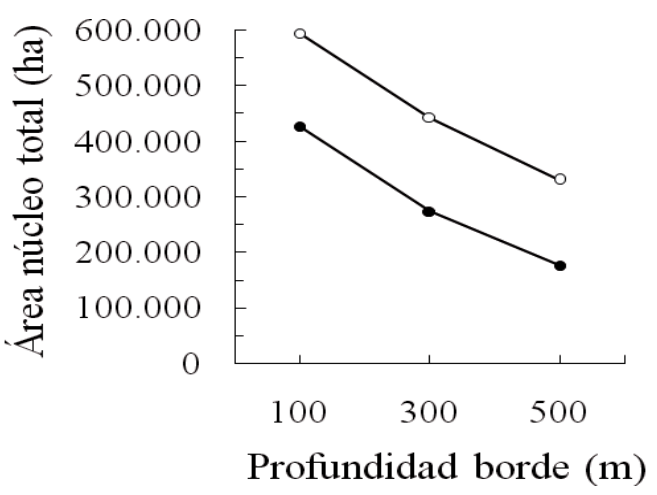

Nota: ( o ) "bosque histórico" (ca. 1900) y en (•) "bosque actual" (1998).

Fuente: Elaboración propia.

\section{Consideraciones finales}

A través del presente trabajo se ha podido reconocer y cuantificar la pérdida y fragmentación del bosque nativo de la cuenca del río Aysén, debido principalmente al uso antrópico del fuego. Esto último concordaría con otro trabajo realizado en la misma área de estudio por Torres-Gómez et al. (2009), donde además se indica que los cambios de uso de suelo allí ocurridos dependieron de factores históricos y culturales específicos. En consecuencia se debe destacar la gran utilidad prestada por la integración de los registros históricos y el Sistema de Información Geográfica (SIG) como herramientas fundamentales en la reconstrucción del paisaje boscoso de Aysén.

Se estima que para la década de 1900 los bosques de la cuenca del río Aysén mantenían una alta pristinidad, calculándose que la zona presentaba alrededor de 675.519 ha de bosque nativo. Se calculó una pérdida de 154.056 ha, equivalentes a un $23 \%$ del bosque nativo, entre los años 1900 y 1998, debido principalmente a los grandes incendios forestales ocurridos a me- 
diados del siglo XX. Los incendios forestales repercutieron directamente en los patrones de distribución del bosque nativo de la cuenca del río Aysén. Su fragmentación se caracterizó por un aumento en el número, densidad y borde total de los fragmentos, además de la disminución del tamaño medio y área núcleo de estos. Los incendios forestales históricos, provocaron la disección de los bosques y no la típica fragmentación parchosa. Finalmente, no se registra una importante pérdida de conectividad entre los fragmentos de bosque nativo presentes en la cuenca del río Aysén.

\section{Referencias bibliográficas}

AGUAYO, M. Uso de los sistemas de información geográfica como herramienta de análisis de fragmentación del bosque nativo. El caso de Biobío. Bosque Nativo, 2001, $\mathrm{N}^{\circ}$ 30, p. 8-13.

ALTAMIRANO, A.; ECHEVERRIA, C. y LARA, A. Efecto de la fragmentación forestal sobre la estructura vegetacional de las poblaciones amenazadas de Legrandia concinna (Myrtaceae) del centro-sur de Chile. Revista Chilena de Historia Natural, 2007, No 80, p. 27- 42 .

ANDRÉN, H. Effects of habitat fragmentation on birds and mammals in landscapes with different proportions of suitable habitat: a review. Oikos, 1994, No 71, p. 355-366.

ARMENTERAS, D.; GAST, F. \& VILLARREAL, H. Andean forest fragmentation and the representativeness of protected natural areas in the eastern Andes, Colombia. Biological Conservation, 2003, N N 113, p. 245256.

ARMESTO, A.; LEÓN, P. y ARROYO, M. Los bosques templados del sur de Chile y Argentina: una isla biogeográfica. En: ARMESTO, A.; VILLAGRÁN, J. y ARROYO, M. C. (eds.). Ecología de los Bosques Nativos de Chile. Santiago: Editorial Universitaria, 1997, p. $71-92$.

ARMESTO, J.; VILLAGRAN, C. y DONOSO, C. Desde la era glacial a al industrial. La historia del bosque templado chileno. Ambiente y desarrollo, 1994, Marzo, p. 66-72.
BOLÓS, M. DE. La evolución o arqueología del paisaje. En: BOLÓS, M. DE. (ed.). Manual de ciencia del paisaje teoría, métodos y aplicaciones. Barcelona: Masson S. A., 1992, p. $191-203$.

BUREL, F. y BAUDRY, J. Ecología del paisaje. Conceptos, métodos y aplicaciones. Madrid: Ediciones Mundi-prensa, 2002.

BUSTAMANTE, R. y GREZ, A. Consecuencias ecológicas de la fragmentación de los bosques nativos. Ambiente y Desarrollo, 1995, vol. 11, No 2, p. 58-63.

CAYUELA, L.; REY-BENAYAS, J. M. \& ECHEVERRIA, C. Clearance and fragmentation of tropical montane forests in the Highlands of Chiapas, Mexico (1975-2000). Forest Ecology and Management, 2006, No 226, p. 208-218.

CHEN, J.; FRANKLIN, J. F. \& SPIES, T. A. Growing-season microclimatic gradients from clearcut edges into old-growth Douglas-Fir forest. Ecogical Applications, 1995, № 5, p. 74-86.

CORPORACIÓN NACIONAL FORESTAL (CONAF); COMISIÓN NACIONAL DEL MEDIO AMBIENTE (CONAMA); BANCO INTERNACIONAL DE RECONSTRUCCIÓN Y FOMENTO (BIRF); UNIVERSIDAD AUSTRAL DE CHILE; PONTIFICIA UNIVERSIDAD CATÓLICA DE CHILE y UNIVERSIDAD CATÓLICA DE TEMUCO. Catastro y Evaluación de los recursos vegetacionales Nativos de Chile. Santiago: CONAF, Informe Nacional con Variables Ambientales, 1999.

CUNILL, P. Geografía de Chile. Santiago: Editorial Universitaria, 1979.

DIRECCIÓN GENERAL DE AGUAS (DGA). Diagnóstico y clasificación de los cursos y cuerpos de agua según objetivos de calidad. Santiago: MOP, Informe Cade-Idepe Consultores en Ingeniería, 2004.

DONOSO, C. Bosques templados de Chile y Argentina: variación, estructura y dinámica. Santiago: Editorial Universitaria, 1993.

DONOSO, P. y OTERO, L. Hacia una definición de país forestal: ¿dónde se sitúa Chile? Bosque, 2005, vol. 26, № 3, p. 5-18. 
ECHEVERRÍA, C.; COOMES, D.; SALAS, J.; REY-BENAYAS, J. M.; LARA, A. \& NEWTON, A. Rapid deforestation and fragmentation of Chilean temperate forest. Biological Conservation, 2006, No 130, p. 181 191.

ECHEVERRÍA, C.; NEWTON, A.; LARA, A.; REY-BENAYAS, J. M. \& COOMES, D. Impacts of forest fragmentation on species composition and forest structure in the temperate landscape of southern Chile. Global Ecology and Biogeography, 2007, N $N^{\circ} 16$, p. 426-439.

ENVIRONMENTAL SYSTEMS RESEARCH INSTITUTE (ESRI). ArcView 3.2. California: ESRI Inc., 1999.

FAHRIG, L. Relative effects of hábitat loss and fragmentation on species extinction. Journal of Wildlife Management, 1997, Nº 61, p. 603-610.

FAHRIG, L. Effects of habitat fragmentation on biodiversity. Annual Review of Ecology, Evolution and Systematics, 2003, N $N^{\circ} 34$, p. 487-515.

FAHRIG, L. \& MERRIAM, G. Conservation of fragmented populations. Conservation Biology, 1994, № 8, p. 50-59.

FLETCHER, R. J.; RIES, J. R. L.; BATTIN, J. \& CHALFOUN, A. The role of habitat area and edge in fragmented landscapes: definitively distinct or inevitably intertwined? Canadian Journal of Zoology, 2007, No 85, p. 1017-1030.

FORMAN, R. T. T. \& GODRON, M. Landscape ecology. New York: John Wiley \& Sons, 1986.

GAJARDO, R. La vegetación natural de Chile: clasificación y distribución geográfica. Santiago: Editorial Universitaria, 1994.

GÓMEZ-LOBO, A. El consumo de leña en el sur de Chile: ¿por qué nos debe preocupar y qué se puede hacer? Ambiente y desarrollo, 2005, vol. 21, No 3, p. 43-77.

GROSSE, A. Visión de Aisén. Expediciones del explorador Juan Augusto Grosse. Santiago: inédito, 1955.
GROSSE, A. Visión de Aisén. Santiago: Author`s Publication, 1974.

IBÁÑEZ, A. La incorporación de Aysén a la vida nacional, 1902-1936. Historia, 1972 1973, No 11, p. 259-378.

INSTITUTO GEOGRÁFICO MILITAR (IGM). Geografía de Chile-Hidrografía (Tomo VIII). Santiago: IGM, 1984.

KELLER, C. La región del hielo continental frente al lago Buenos Aires. Revista Chilena de Historia y Geografía, 1948, No 122, p. 327-331.

LARA, A.; CORTÉS, M. y ECHEVERRÍA, C. Bosques. En: SUNKEL, O. (ed.). Informe país: estado actual del medio ambiente en Chile. Santiago: Centro de Estudios de Políticas Públicas, Universidad de Chile, 2000, p. 131-173.

LAURANCE, W. Do edge effects occur over large spatial scales? Trends in Ecology and Evolution, 2000, No 15, p. 134-135.

LINDENMAYER, D. \& FISCHER, J. Habitat fragmentation and landscape change: an ecological and conservation synthesis. Washington: Isaland Press, 2006.

LÓPEZ-BARRERA, F. Estructura y función en bordes de bosques. Ecosistemas, 2004, vol. 13, No 1, p. 67-77.

LÓPEZ-BARRERA, F.; NEWTON, A. \& MANSON, R. Edge effects in a tropical montane forest mosaic: experimental tests of postdispersal acorn removal. Ecological Research, 2005, No 20, p. 31-40.

MARTINIC, M. De la Trapananda al Aysén. Santiago: Pehuén Editores, 2005.

MCGARIGAL, K.; CUSHMAN, S. A.; NEEL, M. C. \& ENE, E. FRAGSTATS: spatial pattern analysis program for categorical maps. Computer software program produced by the authors at the University of Massachusetts. Amherst: University of Massachusetts, 2002. Disponible en Internet: www.umass.edu/landeco/research/fragstats/fragstats.html

MILLER, A. The climate of Chile. En: SCHWERDTFEGER, W. (ed.). World survey 
of climatology. Amsterdam: Elsevier, 1976, p. 113-145.

MUSTERS, G. Vida entre los patagones. En: FALKNER, T. (ed.). Descripción de la Patagonia. Vol. I. Buenos Aires: Imprenta de Coni Hermanos, 1911.

MYERS, N.; MITTERMELER, R. A.; MITTERMELER, C. G.; DA FONSECA, G. A. B. \& KENT, J. Biodiversity hotspots for conservation priorities. Nature, 2000, $\mathrm{N}^{\circ} 403$, p. 853-858.

ORTEGA, H. y BRÜNING, A. Aisén. Panorama histórico y cultural XI Región. Santiago: Editorial Lom, 2004.

OTERO, L. La huella del fuego. Historia de los bosques nativos poblamiento y cambios en el paisaje en el sur de Chile. Santiago: Pehuén Editores, 2006.

PAUCHARD, A.; AGUAYO, M. y ALABACK, P. Cuantificando la fragmentación del paisaje: las métricas y sus significados ecológicos. En: GREZ, A.; SIMONETTI, J. y BUSTAMANTE, R. (eds.). Biodiversidad en ambientes Fragmentados de Chile: patrones y procesos a diferentes escalas. Santiago: Editorial Universitaria, 2006, p. 41-67.

POMAR, J. La concesión de Aisén y el valle Simpson. (Notas y recuerdos de un viaje de inspección en mayo y junio de 1920). Santiago: Imprenta Cervantes, 1923.

QUINTANILLA, V. Alteraciones del fuego sobre la biodiversidad de bosques templados. El caso del bosque pluvial costero de Chile. Cuadernos geográficos, 2001, № 31, p. 7-21.

QUINTANILLA, V. Estado de recuperación del bosque nativo en una cuenca nordpatagónica de Chile, perturbada por grandes fuegos acaecidos 50 años atrás $\left(44^{\circ}-45^{\circ} \mathrm{S}\right)$. Revista de Geografía Norte Grande, 2008, № 39, p. 73-92.

REICHE, K. Geografía botánica de Chile, 1860-1920. Vol. I. Santiago: Imprenta Universitaria, 1934.

RIES, L.; FLETCHER, R. J.; BATTIN, J. \& SISK, T. D. Ecological responses to habitat edges: mechanisms, models, and variability explained. Annual Review of Ecology. Evolution and Systematics, 2004, № 35, p. 491522.

SAN MARTÍN, J. y DONOSO, C. Estructura florística e impacto antrópico en el bosque maulino de Chile. En: ARMESTO J. J.; VILLAGRÁN, C. y ARROYO, M. K. (eds.). Ecología de los bosques nativos de Chile. Santiago: Editorial Universitaria, 1996, p. 163- 165.

SANTOS, T. y TELLERÍA, J. L. Pérdida y fragmentación del hábitat: efecto sobre la conservación de las especies. Ecosistemas, 2006. № 2, p. 3-12.

SAUNDERS, D. A.; HOBBS, R. J. \& MARGUALES, C. R. Biological consequences of ecosystem fragmentation: a review. Conservation Biology. 1991, № 5, p. 18-32.

SEGURA, A.; HOLMGREN, M.; ANABALÓN, J. \& FUENTES, R. The siginificante of fire intensity in of nine species forming soil seed banks within the Sydney region. Austral Ecology, 1998, № 28, p. 674-683.

SEPÚLVEDA, C.; MOREIRA, A. y VILLARROEL, P. Conservación biológica fuera de las áreas protegidas. Ambiente y Desarrollo, 1997, vol. 13, No 2, p. 48-56.

SEPÚLVEDA, F. La provincia de Aisén. Historia, formación y desarrollo de las regiones que forman la provincia actual de Aisén. Santiago: Imprenta Ravill, 1931.

SIMONETTI, J. \& ARMESTO, J. Conservation of temperate ecosystems in Chile: coarse versus fine-filter approaches. Revista Chilena de Historia Natural, 1991, No 64, p. 615-626.

SIMPSON, E. Esploraciones hechas por la corbeta Chacabuco al mando del capitan de fragata D. Enrique M. Simpson en los archipielagos de las Guaitecas, Chonos i Taitao. Anuario Hidrográfico de la Marina de Chile, $1875, N^{\circ} 1$, p. 3-147.

STAUS, N.; STRITTHOLT, J.; DELLASALA, D. \& ROBINSON, R. Rate and patterns of forest disturbance in the Klamath-Siskiyou ecoregion, USA between 1972 and 1992. Landscape Ecology, 2002, № 17, p. 455-470. 
STEFFEN, H. Viajes de exploración i estudio en la Patagonia occidental 1892-1902 por el Dr. Hans Steffen. Vol. II. Santiago: Imprenta Cervantes, 1909.

TORRES-GÓMEZ, M.; DELGADO, L.; MARÍN, V. y BUSTAMANTE, R. Estructura del paisaje a lo largo de gradientes urbano- rurales en la cuenca del río Aisén (Región de Aisén, Chile). Revista Chilena de Historia Natural, 2009, vol. 82, № 1, p. 73-82.

ZENG, H. \& WU, X. Utilities of edge-based metrics for studying landscape fragmentation. Computers. Environment and Urban Systems, 2005, vol. 29, № 2, p. 159-178. 